\title{
Performance of a cooperative multiplexing scheme with opportunistic user and relay selection over Rayleigh fading channels
}

\author{
Dileep Kumar Verma and Shankar Prakriya*
}

\begin{abstract}
In this article, we analyze the performance of a cooperative spatial multiplexing scheme using amplify and forward relays over Rayleigh fading channels. We propose a simple user and relay selection scheme, and demonstrate that gains in spectral efficiencies are possible by such opportunistic selection. Using a bound on the outage performance, we develop a power control mechanism. We develop a bound on the ergodic rate of the proposed scheme. Computer simulation results are presented to demonstrate the performance of the scheme, and to validate the analytical expressions.
\end{abstract}

\section{Introduction}

Several new standards for cellular communication are now incorporating amplify and forward (AF) or decode and forward (DF) relays [1]. Cooperative communication protocols have the ability to exploit these relays to harness the spatial features of the network for reliable data transmissions [1-3]. A variety of protocols has been proposed to obtain higher spectral efficiencies, better outage performance, or to save transmitter power. Typically, the relays are half-duplex due to practical constraints, and this leads to loss of spectral efficiency. Various protocols have been proposed to overcome this limitation, and their performance has been analyzed [4].

It is well known that enormous gains in spectral efficiencies are possible through frequency reuse by exploiting the spatial features of the network [5-8]. Yomo and Carvalho [5] discuss the spectral efficiency improvement of the cellular system where two mobile subscribers uplink their respective data to base station (BS) while an AF relay facilitates communication to a shadowed subscriber. For downlink transmission, a similar type of spectrally efficient scheme to obtain gain in spectral efficiency for two users is proposed in [6]. Thai and Popovski [7] proposed an alternative to two-way relaying, and use a half-duplex

${ }^{*}$ Correspondence: shankar@ee.iitd.ac.in

Department of Electrical Engineering, Indian Institute of Technology, Delhi, India relay to obtain significant improvement in spectral efficiency. Cooperative multiplexing protocols [9-11] proposed in recent years help realize these promised gains through frequency reuse. In addition, they also help to overcome the loss in spectral efficiency due to half-duplex nature of practical relays. In [10], a two-user cooperative multiplexing and scheduling (CMS) protocol using AF relays has been proposed, where a BS multiplexes the data of two selected users. In the first phase, the BS transmits the signal intended for the second user to the relay. In the second phase, the BS transmits the signal intended for the first user, while the relay retransmits the signal intended for the second user. In [11], interference cancellation and precoding techniques are proposed (assuming DF relays) to deal with the interference due to simultaneous transmissions in the second phase. This interference (in the second phase) makes the study of power distribution between the BS and the RS important. While Shi et al. [10] do not address this issue, an optimum power distribution is derived in [11] for the case when DF relays are utilized. In both $[10,11]$, the focus is on the achievable sum data rates, though some simplified outage analysis is also presented assuming Rayleigh fading channels. Performance with these protocols is limited by the link between the BS and the relay. Although CMS protocols employing relay selection are consequently of interest, they have not been considered so far. To the best of the authors' knowledge, outage and ergodic rate performance of CMS protocols

\section{是 Springer}

(C) 2012 Verma and Prakriya; licensee Springer. This is an Open Access article distributed under the terms of the Creative

Commons Attribution License (http://creativecommons.org/licenses/by/2.0), which permits unrestricted use, distribution, and reproduction in any medium, provided the original work is properly cited. 
with AF relays have not been analyzed for Rayleigh fading channels.

In this article, we consider an opportunistic user and relay selection-based CMS system that enables the BS to multiplex data to a (selected) user, and another (selected) user in the shadowed region. AF relays are utilized for transmission to the user in the shadowed region. A sub-optimal distributed users' selection scheme is proposed. For relay selection the sub-optimal scheme [12] is employed to facilitate distributed implementation. However, we propose a sub-optimal selection strategy based on [12] for the CMS network, in which the node is selected that maximizes the gain of the corresponding channel for desired signal, unlike the optimal node selection scheme in which interference channel should also be considered. Assuming Rayleigh fading channels, an analysis of the outage probability and ergodic performance of the user(s) and relay selection scheme-based CMS system is presented. Since the exact analysis is mathematically intractable, we present bounds of outage and ergodic performances. Moreover, we develop a power control strategy for the second phase of the multiplexing scheme. In summary, the contributions of the article are as follows:

1. We demonstrate that a simple user and relay selection scheme can be implemented in a distributed manner and yield good performance.

2. Unlike other work on cooperative multiplexing [9-11], we consider both user and relay selection. Also, we consider AF relays, analysis for which is complicated by the noise amplification in cooperative multiplexing scenarios.

3. We evolve a simple power control scheme for the second phase of the multiplexing scheme.

The rest of the article is organized as follows. The system model is described in "System model description" section. In "Outage performance \& power control" section, a power control scheme is discussed based on a bound on the outage probability. A bound for the ergodic sum-rate is presented in "Ergodic sum-rate" section. Performance of the proposed protocol is analyzed through computer simulations in "Numerical and simulation results" section, where the derived analytical expressions are validated. Conclusions are drawn in "Conclusion" section.

Notations: The probability density function of gain $\left(|h|^{2}\right)$ of Rayleigh fading channel coefficient $(h)$ is given by $f_{|h|^{2}}(x)=\exp (-x / \Omega) / \Omega$ and cumulative distribution function $\operatorname{Pr}\left(|h|^{2} \leq x\right)=1-\exp (-x / \Omega)$ where $\Omega=$ $\mathbb{E}\left[|h|^{2}\right]$, and $\mathbb{E}[\cdot]$ denotes expectation of random variables. $\Gamma(m)=\int_{0}^{\infty} t^{m-1} e^{-t} d t$ is the Gamma function, and $\mathrm{E}_{n}(x)\left(=\int_{1}^{\infty} \frac{\exp (-x t)}{t^{n}} d t\right)$ represents the exponential integral of order $n$.

\section{System model description}

We consider a cellular cooperative communication system as depicted in Figure 1. It consists of a BS with $Q$ users, and $L$ dedicated distributed relays (RS) of the channel-assisted AF type. Each node is equipped with single antenna, and operates in a half-duplex mode. The $Q$ users are grouped into two clusters $(\mathcal{A}$ and $\mathcal{B})$ of $M$ and $N$ so that $Q=M+N$. The $M$ users (in cluster $\mathcal{A}$ ) and $L$ relays (in cluster $\mathcal{L}$ ) have strong average channel link with the BS, while the $N$ users in cluster $\mathcal{B}$ are assumed to be in a shadowed region with a comparatively weak channel link to the BS. Relays are utilized to enable the BS to ensure data reliability for users in set $\mathcal{B}$. Using a sub-optimal scheme, one user $m \in \mathcal{A}$ and another user $n \in \mathcal{B}$ are selected along with one relay $l \in \mathcal{L}$. The selection scheme is discussed later in this section. In the considered CMS system, only two time slots are required for the BS to transmit data to two different users despite the use of relays. In the first time-slot, the BS transmits data of user $n \in \mathcal{B}$ to the relay. In the second time-slot, the BS and one opportunistically selected relay $l \in \mathcal{L}$ spatial multiplex the data of user $n \in \mathcal{B}$ and one selected user $m \in \mathcal{A}$.

We assume that the fading channels (from any node) to nodes in each cluster are statistically independent and identically distributed (i.i.d.). The Rayleigh fading channel coefficients are denoted by $h_{j, k}$ where source $j \in$ $\{\mathrm{BS}, l\}$, destination $k \in\{l, m, n\}, l \in\{1,2, \ldots, L\}, m \in$ $\{1,2, \ldots, M\}$, and $n \in\{1,2, \ldots N\}$. The inter node distance (nodes in separate cluster) is approximated to inter cluster distance, as nodes in cluster are close to each other as compared to distance between clusters. Therefore, the average channel gains of various links are denoted by $\Omega_{j, k}=\mathbb{E}\left[\left|h_{j, k}\right|^{2}\right]=\frac{1}{d_{j, k}^{\alpha}}$, where $d_{j, k}$ is the distance between nodes/clusters $j$ and $k$, and $\alpha$ is the pathloss exponent. Note, we use subscripts $\mathrm{BS}, \mathrm{A}, \mathrm{R}$, and $\mathrm{B}$ for alternatively

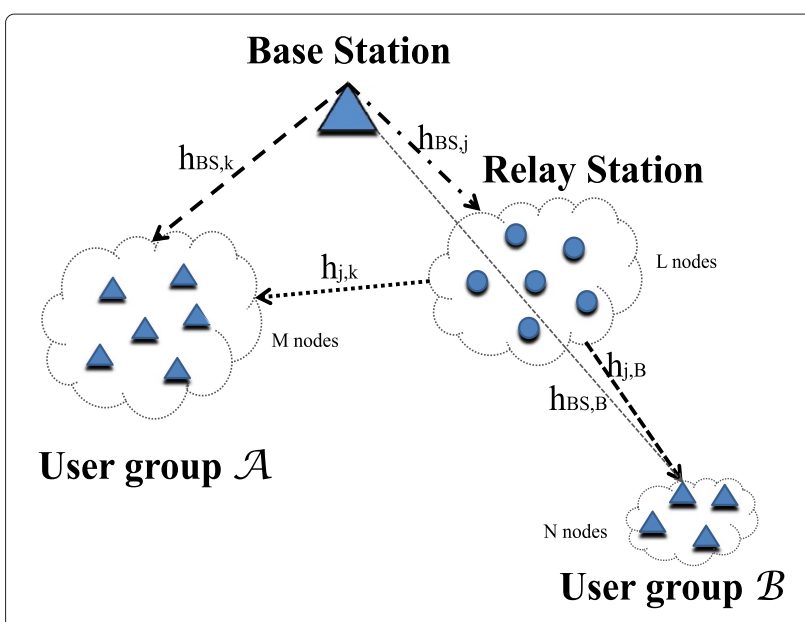

Figure 1 System model. 
representing the BS, selected user from $\mathcal{A}$, selected relay from RS, and selected user from $\mathcal{B}$, respectively.

In the first phase (time slot), the BS broadcasts unit energy symbols $\left(s_{\mathrm{B}}\right)$ with power $P$ meant for user $n \in$ $\mathcal{B}$. The $l$ th relay and $m$ th user receive symbols $y_{l}=$ $\sqrt{P} h_{\mathrm{BS}, l} s_{\mathrm{B}}+w_{l}$ and $y_{m}^{\mathrm{I}}=\sqrt{P} h_{\mathrm{BS}, m} s_{\mathrm{B}}+w_{m}^{\mathrm{I}}$, respectively, where superscript "I" represents the phase number (first, in this case), and $w_{l}$ and $w_{m}^{\mathrm{I}}$ are zero mean additive white Gaussian noises with variance $\sigma_{w}^{2}$ at the $l$ th relay and $m$ th user, respectively. User $n \in \mathcal{B}$ in the shadowed region ignores the weak signal from the BS in this phase. The $m$ th user of cluster $\mathcal{A}$ detects the symbols $s_{\mathrm{B}}$, and utilizes it in the subsequent phase to mitigate interference caused by simultaneous transmission from BS and RS.

In second time slot, to exploit spatial multiplexing, the BS transmits symbols $s_{\mathrm{A}}$ of $m$ th user with transmitting power $P_{\mathrm{BS}}$, while the $l$ th relay simultaneously forwards the amplified version of the signal meant for user $n \in \mathcal{B}$ with power $P_{\mathrm{RS}}$. The manner in which $P_{\mathrm{BS}}$ and $P_{\mathrm{RS}}$ are determined to minimize the outage probability is discussed in "Outage performance \& power control" section. User $n \in$ $\mathcal{B}$ receives symbols $y_{n}=\sqrt{P_{\mathrm{RS}}} h_{l, n} \beta y_{l}+\sqrt{P_{\mathrm{BS}}} h_{\mathrm{BS}, n} s_{\mathrm{A}}+w_{n}$ using $l$ th relay, where $\beta=1 /\left(\sqrt{P\left|h_{\mathrm{BS}, l}\right|^{2}+\sigma_{w}^{2}}\right)$ and $w_{n}$ is zero mean additive white Gaussian noise with variance $\sigma_{w}^{2}$. The signal-to-interference-plus noise ratio (SINR) at user $n \in \mathcal{B}$ via the $l$ th relay is given by

$$
\begin{aligned}
\gamma_{n, l} & =\frac{\gamma\left|h_{\mathrm{BS}, l}\right|^{2} \gamma_{\mathrm{RS}}\left|h_{l, n}\right|^{2}}{\gamma_{\mathrm{RS}}\left|h_{l, n}\right|^{2}+\mathcal{I}_{n}\left(\gamma\left|h_{\mathrm{BS}, l}\right|^{2}+1\right)} \\
& =\frac{\gamma\left|h_{\mathrm{BS}, l}\right|^{2}\left(\gamma_{\mathrm{RS}}\left|h_{l, n}\right|^{2} / \mathcal{I}_{n}\right)}{\gamma\left|h_{\mathrm{BS}, l}\right|^{2}+\left(\gamma_{\mathrm{RS}}\left|h_{l, n}\right|^{2} / \mathcal{I}_{n}\right)+1} \\
& \approx \frac{\gamma\left|h_{\mathrm{BS}, l}\right|^{2}\left(\gamma_{\mathrm{RS}}\left|h_{l, n}\right|^{2} / \mathcal{I}_{n}\right)}{\gamma\left|h_{\mathrm{BS}, l}\right|^{2}+\left(\gamma_{\mathrm{RS}}\left|h_{l, n}\right|^{2} / \mathcal{I}_{n}\right)},
\end{aligned}
$$

where $\gamma_{\mathrm{RS}}=P_{\mathrm{RS}} / \sigma_{w}^{2}, \mathcal{I}_{n}=\gamma_{\mathrm{BS}}\left|h_{\mathrm{BS}, n}\right|^{2}+1, \gamma_{\mathrm{BS}}=\frac{P_{\mathrm{BS}}}{\sigma_{w}^{2}}$, and the SNR $\gamma=\frac{P}{\sigma_{w}^{2}}$. The last expression is obtained by neglecting the 1 in the denominator. This sort of approximation has widely been used in literature, and the approximate $\gamma_{n, l}$ so obtained has been shown to be indistinguishable from the true value at all SNRs $\gamma$. Using the well-known bounds on the harmonic mean [13], we can then see that the SINR is bounded by:

$$
\begin{aligned}
& \frac{1}{2} \min \left(\gamma\left|h_{\mathrm{BS}, l}\right|^{2}, \gamma_{\mathrm{RS}}\left|h_{l, n}\right|^{2} / \mathcal{I}_{n}\right) \\
& \quad \leq \gamma_{n, l}<\min \left(\gamma\left|h_{\mathrm{BS}, l}\right|^{2}, \gamma_{\mathrm{RS}}\left|h_{l, n}\right|^{2} / \mathcal{I}_{n}\right) .
\end{aligned}
$$

Accordingly, we note that the upper bound $\gamma_{n, l}^{\mathrm{UB}}$ on the SNR is given by

$$
\gamma_{n, l}^{\mathrm{UB}}=\min \left(\gamma\left|h_{\mathrm{BS}, l}\right|^{2}, \gamma_{\mathrm{RS}}\left|h_{l, n}\right|^{2} / \mathcal{I}_{n}\right) .
$$

It is clear from (1) and (2) that relay selection (choice $l$ ) plays an important role in determining performance. It is noted that the influence of interference due to the term $\mathcal{I}_{n}$ can readily be minimized independently by minimizing $\mathcal{I}_{n}$. For this reason, it is proposed to choose $n$ to minimize $I_{n}$. This is discussed in detail in "Outage performance \& power control" Section. Estimation of the channel gains, and selection of users $m^{\star}, n^{\star}$, and relay $l^{\star}$ is facilitated by a training phase that precedes the actual data transmission. Ideally, the best users and best relay should be selected jointly so as to minimize the outage probability. However, such joint selection, though ideal, is difficult to implement. Note that this users and relay selection scheme are important in a distributed manner. We therefore propose to use the sub-optimal scheme such that a distributed timer-based scheme for cooperative communication network is used to opportunistically select the best relay node as given in $[12,14]$. Making use of the fact that the term $\mathcal{I}_{n}$ is common to all $\gamma_{n, l}$ (for different relays to $n$th node), we directly use [12] for the selection of the best relay $\left(l^{\star}=\arg \max _{l \in \mathcal{L}} \gamma_{n, l}\right)$ as explicit mechanism of selection is already discussed in [12]. For ease of presentation, we first consider the single user set $\mathcal{B}$, i.e., $N=1$, and later in "Outage performance \& power control" and "Ergodic sum-rate" sections show how the case of general $N$ can be accommodated.

Therefore, SINR of user in $\mathcal{B}$ using the opportunistically selected relay $\left(l^{\star}\right)$ is given by

$$
\gamma_{n, l^{\star}}=\max _{l \in \mathcal{L}} \gamma_{n, l}
$$

With the chosen relay, the $m$ th user receives $y_{m}^{\mathrm{II}}=$ $\sqrt{P_{\mathrm{BS}}} h_{\mathrm{BS}, m} x s_{\mathrm{A}}+\sqrt{P_{\mathrm{RS}}} h_{l^{\star}, m} \beta y_{l^{\star}}+w_{m}^{\mathrm{II}}$ in the second phase, where $w_{m}^{\text {II }}$ is additive noise of variance $\sigma_{w}^{2}$. The interfering symbols $s_{\mathrm{B}}$ at users in cluster $\mathcal{A}$ can be cancelled using the symbols decoded in the first phase [11].

After cancellation, the resultant signal of the $m$ th user is $\bar{y}_{m}^{\mathrm{II}}=\sqrt{P_{\mathrm{BS}}} h_{\mathrm{BS}, m} s_{\mathrm{A}}+\sqrt{P_{\mathrm{RS}}} h_{l^{\star}, m} \beta w_{l^{\star}}+w_{m}^{\mathrm{II}}$. It should be emphasized that despite cancellation of the interference, the noise amplified by the AF relay degrades the SNR at the $m$ th user. The SNR with interference cancellation for the $m$ th user is

$$
\begin{aligned}
\gamma_{m} & =\frac{\gamma_{\mathrm{BS}}\left|h_{\mathrm{BS}, m}\right|^{2}\left(\gamma\left|h_{\mathrm{BS}, l^{\star}}\right|^{2}+1\right)}{\gamma\left|h_{\mathrm{BS}, l^{\star}}\right|^{2}+\gamma_{\mathrm{RS}}\left|h_{l^{\star}, m}\right|^{2}+1}, \\
& =\gamma_{\mathrm{BS}}\left|h_{\mathrm{BS}, m}\right|^{2} \Gamma,
\end{aligned}
$$

where $\Gamma=\frac{\gamma\left|h_{\mathrm{B}, l^{\star}}\right|^{2}+1}{\gamma\left|h_{\mathrm{BS}, l^{\star}}\right|^{2}+\gamma_{\mathrm{RS}}\left|h_{l^{\star}, m}\right|^{2}+1}$. In (5), it is readily seen that SNR of $m$ th user is degraded by the factor $\Gamma$ (that takes values between 0 and 1). It is emphasized that the 
ratio $\Gamma$ tends to unity as number of relay increases because BS to $l^{\star}$ channel gain maximizes. Selection of the best user from cluster $\mathcal{A}$ should therefore be made to maximize this quantity. Clearly, the SNR in (5) is bounded as

$$
\gamma_{m} \leq \gamma_{\mathrm{BS}}\left|h_{\mathrm{BS}, m}\right|^{2} \triangleq \gamma_{m}^{\mathrm{UB}}
$$

The SNR of opportunistically selected node $\left(m^{\star}\right)$ is given as

$$
\gamma_{m^{\star}}=\max _{m \in\{1,2, \ldots, M\}} \gamma_{m}
$$

It is noted that the selection of user $m \in \mathcal{A}$ has to take into consideration the degradation in SNR due to noise amplification at the relay. This degradation performance is a consequence of the use of AF relays. However, AF relays possess several advantages in implementations, and introduce less delay as compared to DF relays.

Relay selection in (4) ensures that $h_{\mathrm{BS}, l^{\star}}$ is large. Further, user selection using (7) ensures that $\Gamma$ in (5) is close to unity. For this reason, when $L>1$ and $M>1, \gamma_{m^{\star}}^{\mathrm{UB}}$ is almost always close to $\gamma_{m^{\star}}$.

Using the relay selection as per (4), and user selection as per (7), we analyze the performance of the cooperative multiplexing scheme in the following sections.

\section{Outage performance \& power control}

In this section, we investigate the outage performance of the considered system, and demonstrate how powers $P_{\mathrm{BS}}$ and $P_{\mathrm{RS}}$ can be selected to minimize the outage performance (which is a relevant QoS parameter in the cellular scenario).

Assuming a target rate of $\mathcal{R}$ for the users, the system can be said to be in outage if either of the users fails to attain the target SNR $\gamma_{\text {th }}=2^{2 \mathcal{R}}-1$. Hence, the overall outage probability $p_{o}$ of the system is given by

$$
p_{o}=\operatorname{Pr}\left\{\min \left(\gamma_{m^{\star}}, \gamma_{n, l^{\star}}\right)<\gamma_{\mathrm{th}}\right\}
$$

Since, the signaling mechanism signals to two users together, such an overall outage probability is more meaningful than a single-user outage, and has been used by other authors in the context of cooperative multiplexing $[10,11]$. From (1) and (5), it can be seen that the SNRs at the two terminals $\gamma_{m^{\star}}$ and $\gamma_{n, l^{\star}}$ are statistically dependent, making analysis of the exact outage performance intractable. In what follows, we derive a lower bound $p_{o}^{\mathrm{LB}}\left(=\operatorname{Pr}\left\{\min \left(\gamma_{m^{\star}}^{\mathrm{UB}}, \gamma_{n, l^{\star}}^{\mathrm{UB}}\right) \leq \gamma_{\mathrm{th}}\right\}\right)$ on the outage probability.

To this end, we use the fact that

$\operatorname{Pr}\left\{\min \left(\gamma_{m^{\star}}, \gamma_{n, l^{\star}}\right) \leq \gamma_{\mathrm{th}}\right\} \geq \operatorname{Pr}\left\{\min \left(\gamma_{m^{\star}}^{\mathrm{UB}}, \gamma_{n, l^{\star}}^{\mathrm{UB}}\right) \leq \gamma_{\mathrm{th}}\right\}=p_{o}^{\mathrm{LB}}$, where $\gamma_{m^{\star}}^{\mathrm{UB}}$ and $\gamma_{n, l^{\star}}^{\mathrm{UB}}$ are given by (6) and (2), respectively. Making use of the statistical independence of $\gamma_{m^{\star}}^{\mathrm{UB}}$ and $\gamma_{n, l^{\star}}^{\mathrm{UB}}$, we can write

$$
\begin{aligned}
p_{o}^{\mathrm{LB}}=1 & -\left[1-\prod_{m=1}^{M} \operatorname{Pr}\left(\gamma_{\mathrm{BS}}\left|h_{\mathrm{BS}, m}\right|^{2} \leq \gamma_{\mathrm{th}}\right)\right] \\
\times & {\left[1-\operatorname{Pr}\left(\gamma_{n, l^{\star}}^{\mathrm{UB}} \leq \gamma_{\mathrm{th}}\right)\right] . }
\end{aligned}
$$

Since $\left|h_{\mathrm{BS}, m}\right|^{2}$ is exponentially distributed, it is readily seen that

$$
\operatorname{Pr}\left(\gamma_{\mathrm{BS}}\left|h_{\mathrm{BS}, m}\right|^{2} \leq \gamma_{\mathrm{th}}\right)=1-\exp \left(-\gamma_{\mathrm{th}} /\left(\gamma_{\mathrm{BS}} \Omega_{\mathrm{BS}, \mathrm{A}}\right)\right)
$$

where $\Omega_{\mathrm{BS}, \mathrm{A}}$ is an average channel gain between BS and cluster $\mathcal{A}$.

It is clear from (3) that the CDF of $\gamma_{n, l^{\star}}^{\mathrm{UB}}$ can be obtained using

$$
\operatorname{Pr}\left(\gamma_{n, l^{\star}}^{\mathrm{UB}} \leq \gamma_{\mathrm{th}}\right)=\int_{1}^{\infty} \operatorname{Pr}\left(\gamma_{n, l^{\star}}^{\mathrm{UB}} \leq \gamma_{t h} \mid \mathcal{I}_{n}\right) f_{\mathcal{I}_{n}}(i) d i,
$$

where $f_{\mathcal{I}_{n}}$ (.) denotes the PDF of $\mathcal{I}_{n}=\gamma_{\mathrm{BS}}\left|h_{\mathrm{BS}, n}\right|^{2}+1$ (note that $\mathcal{I}_{n}$ takes values between 1 and $\infty$ ). Using the fact that channel gains $\left|h_{\mathrm{BS}, l}\right|^{2},\left|h_{l, n}\right|^{2}$ and $\left|h_{\mathrm{BS}, n}\right|^{2}$ are independent and exponentially distributed, it can readily be shown that

$$
\begin{aligned}
\operatorname{Pr}\left(\gamma_{n, l^{\star}}^{\mathrm{UB}} \leq \gamma_{\mathrm{th}}\right)=1 & -\sum_{l=1}^{L}(-1)^{l-1}\left(\begin{array}{c}
L \\
l
\end{array}\right) \\
& \times \frac{\exp \left(-\gamma_{\mathrm{th}} l\left(\frac{1}{\gamma \Omega_{\mathrm{BS}, \mathrm{R}}}+\frac{1}{\gamma_{\mathrm{RS}} \Omega_{\mathrm{R}, \mathrm{B}}}\right)\right)}{\frac{l \gamma_{\mathrm{th}} \gamma_{\mathrm{BS}} \Omega_{\mathrm{BS}, \mathrm{B}}}{\gamma_{\mathrm{RS}} \Omega_{\mathrm{R}, \mathrm{B}}}+1} .
\end{aligned}
$$

From (12), it is notable that $\operatorname{Pr}\left(\gamma_{n, l^{\star}}^{\mathrm{UB}} \leq \gamma_{\text {th }}\right) \rightarrow$ $\left(1-e^{-\gamma_{\mathrm{th}}\left(\frac{1}{\gamma \Omega_{\mathrm{B}, \mathrm{R}}}+\frac{1}{\gamma_{\mathrm{RS}} \Omega_{\mathrm{R}, \mathrm{B}}}\right)}\right)^{L}$ as average interference channel gain $\Omega_{\mathrm{BS}, \mathrm{B}} \rightarrow 0$. This situation occurs as distance between BS and user $n \in \mathcal{B}$ increases. After substituting (10) and (12) in (9), we can obtain $p_{o}^{\mathrm{LB}}$ as

$$
\begin{aligned}
p_{o}^{\mathrm{LB}}= & -\left[1-\left(1-\exp \left(-\frac{\gamma_{\mathrm{th}}}{\gamma_{\mathrm{BS}} \Omega_{\mathrm{BS}, \mathrm{A}}}\right)\right)^{M}\right] \\
\times & {\left[1-\left[1-\sum_{l=1}^{L}(-1)^{l-1}\left(\begin{array}{c}
L \\
l
\end{array}\right)\right.\right.} \\
& \left.\left.\times \frac{\exp \left(-\gamma_{\mathrm{th}} l\left(\frac{1}{\gamma \Omega_{\mathrm{BS}, \mathrm{R}}}+\frac{1}{\gamma_{\mathrm{RS}} \Omega_{\mathrm{R}, \mathrm{B}}}\right)\right)}{\frac{l \gamma_{\mathrm{th}} \gamma_{\mathrm{BS}} \Omega_{\mathrm{BS}, \mathrm{B}}}{\gamma_{\mathrm{RS}} \Omega_{\mathrm{R}, \mathrm{B}}}+1}\right]\right] .
\end{aligned}
$$

In the first phase, no knowledge of the channels to the relays is assumed so that the BS transmits with full power. 
In the second phase, both the BS and the relay transmit simultaneously resulting in interference at both users $m \in$ $\mathcal{A}$ and $n \in \mathcal{B}$ (due to the use of AF). In such interference channels, power control is of great significance. Though no outage expression is possible due to intractability, the bound in (13) can be used to perform power allocation. To this end, we assume that $P_{\mathrm{BS}}=\zeta_{\mathrm{BS}} P$ and $P_{\mathrm{RS}}=\left(1-\zeta_{\mathrm{BS}}\right) P$ so that $P_{\mathrm{BS}}+P_{\mathrm{RS}}=P$, where $\zeta_{\mathrm{BS}}$ lies between 0 and 1 . The power allocation factor $\zeta_{\mathrm{BS}}^{\star}$ can then be obtained from

$$
\zeta_{\mathrm{BS}}^{\star}=\min _{\zeta_{\mathrm{BS}}} p_{o}^{\mathrm{LB}}
$$

through numerical techniques. Note that this requires knowledge of the channel variances only.

\section{Special case}

We now discuss the case when $N>1$ so that user selection is applied for users in set $\mathcal{B}$. It is clear from (1) that the SNR of user $n \in \mathcal{B}$ can be maximized by limiting the interference term $\mathcal{I}_{n}$. This implies that user in set $\mathcal{B}$ can be selected simply by

$$
\mathcal{I}_{n^{\star}}=\min _{n \in \mathcal{B}}\left|h_{\mathrm{BS}, n}\right|^{2}
$$

Interference to user $n \in \mathcal{B}$ because of the signal transmission by the BS in the second phase can similarly be dealt with by user selection in set $\mathcal{B}$. Statistically, the probability density function of the interference power $\mathcal{I}_{n^{\star}}=$ $\gamma_{\mathrm{BS}}\left|h_{\mathrm{BS}, n^{\star}}\right|^{2}+1$ with the help of order statistics [15] is given as

$$
f_{\mathcal{I}_{n^{\star}}}(i)=\frac{1}{\gamma_{\mathrm{BS}} \Omega_{\mathrm{BS}, \mathrm{B}}^{\prime}} \exp \left(-i /\left(\gamma_{\mathrm{BS}} \Omega_{\mathrm{BS}, \mathrm{B}}^{\prime}\right)\right), \quad 1 \leq i<\infty
$$

where $\Omega_{\mathrm{BS}, \mathrm{B}}^{\prime}=\frac{\Omega_{\mathrm{BS}, \mathrm{B}}}{N}$ and $\Omega_{\mathrm{BS}, \mathrm{B}}$ is average channel gain from BS to user in set $\mathcal{B}$. From (15), it is interesting to note that such type of selection directly reduces the channel gain variance by the degree of freedom in selection of user in $\mathcal{B}$. Consequently, the overall outage probability of the system is given by

$$
\begin{aligned}
p_{o}^{\mathrm{LB}}=1 & -\left[1-\left(1-\exp \left(-\frac{\gamma_{\mathrm{th}}}{\gamma_{\mathrm{BS}} \Omega_{\mathrm{BS}, \mathrm{A}}}\right)\right)^{M}\right] \\
\times & {\left[1-\left[1-\sum_{l=1}^{L}(-1)^{l-1}\left(\begin{array}{l}
L \\
l
\end{array}\right)\right.\right.} \\
& \left.\left.\times \frac{\exp \left(-\gamma_{\mathrm{th}} l\left(\frac{1}{\gamma \Omega_{\mathrm{BS}, \mathrm{R}}}+\frac{1}{\gamma_{\mathrm{RS}} \Omega_{\mathrm{R}, \mathrm{B}}}\right)\right)}{\frac{l \gamma_{\mathrm{th}} \gamma_{\mathrm{BS}} \Omega_{\mathrm{BS}, \mathrm{B}}^{\prime}}{\gamma_{\mathrm{RS}} \Omega_{\mathrm{R}, \mathrm{B}}}+1}\right]\right] .
\end{aligned}
$$

It can be seen that as $N$ becomes large, the outage performance improves and outage probability does not saturate as $N \rightarrow \infty$.

\section{Ergodic sum-rate}

In this section, we develop a bound on the ergodic rate, and show that significant improvement in data rates is possible with the increase in $L, M$, and $N$. As in "Outage performance \& power control" section, we first discuss the case when $N=1$, and discuss the case of user selection in user set $\mathcal{B}$ as a special case. With the user and relay selection discussed, the sum rate of the two users $\mathcal{R}_{\text {sum }}$ is given by

$$
\begin{aligned}
\mathcal{R}_{\text {sum }} & =\mathcal{R}_{\mathrm{A}}+\mathcal{R}_{\mathrm{B}}, \\
& =\frac{1}{2} \log _{2}\left(1+\gamma_{m^{\star}}\right)+\frac{1}{2} \log _{2}\left(1+\gamma_{n, l^{\star}}\right),
\end{aligned}
$$

where subscripts а and в represent user $m \in \mathcal{A}$ and $n \in \mathcal{B}$, respectively. We use the bounds for $\gamma_{n, l}$ in (2) to obtain the lower bound of the ergodic sum-rate. Using the fact that $\frac{1}{2} \min \left(\gamma\left|h_{\mathrm{BS}, l}\right|^{2}, \gamma_{\mathrm{RS}}\left|h_{l, n}\right|^{2} / \mathcal{I}_{n}\right) \leq \gamma_{n, l}<$ $\min \left(\gamma\left|h_{\mathrm{BS}, l}\right|^{2}, \gamma_{\mathrm{RS}}\left|h_{l, n}\right|^{2} / \mathcal{I}_{n}\right)$, we can bound the rate of user $n \in \mathcal{B}$. For user $m \in \mathcal{A}$, we need a lower bound on the SNR. To this end, we proceed as follows. We first use a very tight approximation in (5) by assuming that $\gamma\left|h_{\mathrm{BS}, l^{\star}}\right|^{2} \gg 1$ to get $\gamma_{m^{\star}}$ :

$$
\gamma_{m^{\star}} \approx \max _{m \in\{1,2, \cdots, M\}} \gamma_{\mathrm{BS}}\left|h_{\mathrm{BS}, m}\right|^{2} \frac{\gamma\left|h_{\mathrm{BS}, l^{\star}}\right|^{2}}{\gamma\left|h_{\mathrm{BS}, l^{\star}}\right|^{2}+\gamma_{\mathrm{RS}}\left|h_{l^{\star}, m}\right|^{2}} .
$$

Such assumptions are commonly made in analysis of cooperative systems, it can be verified that the CDF of the RHS is indistinguishable from that of $\gamma_{\mathrm{A}, m^{\star}}$. Since, the channel gain $\gamma\left|h_{\mathrm{BS}, l^{\star}}\right|^{2} \geq$ $\max _{l \in\{1,2} \min \left(\gamma\left|h_{\mathrm{BS}, l}\right|^{2}, \gamma_{\mathrm{RS}}\left|h_{l, n}\right|^{2}\right) \triangleq Z$, we use the lower bound of $\gamma\left|h_{\mathrm{BS}, l^{\star}}\right|^{2}$ to simplify the analysis. Note that $\frac{\gamma\left|h_{\mathrm{BS}, l^{\star}}\right|^{2}}{\gamma\left|h_{\mathrm{BS}, l^{\star}}\right|^{2}+\gamma_{\mathrm{RS}}\left|h_{l^{\star}, m}\right|^{2}}$ is a strictly increasing function of $\gamma\left|h_{\mathrm{BS}, l^{\star}}\right|^{2}$. Using this, it can be seen that:

$$
\gamma_{m^{\star}} \gtrsim \tilde{\gamma}_{m^{\star}}=\max _{m \in\{1,2, \cdots, M\}} \gamma_{\mathrm{BS}}\left|h_{\mathrm{BS}, m}\right|^{2} \frac{Z}{Z+\gamma_{\mathrm{RS}}\left|h_{l^{\star}, m}\right|^{2}}
$$

Basically, by increasing number of relays $(L)$ and number of user in cluster $\mathcal{A}(M)$, the ergodic rate of corresponding user is improved. However, increasing $L$ minimizes the noise at user in $\mathcal{A}$ as $Z$ increases, thus the ergodic sum-rate is improved. The detailed interplay between $L$ and $M$ can numerically be viewed in the numerical results section. The CDF of $\tilde{\gamma}_{m^{\star}}$ in (19) is given by

$$
\begin{aligned}
\operatorname{Pr}\left(\tilde{\gamma}_{m^{*}} \leq y\right)= & 1-\sum_{m=1}^{M} \sum_{l=1}^{L} \sum_{i=0}^{m}(-1)^{m+l+i-2}\left(\begin{array}{c}
M \\
m
\end{array}\right)\left(\begin{array}{c}
L \\
l
\end{array}\right)\left(\begin{array}{c}
m \\
i
\end{array}\right) y l C D \\
& \times \exp \left(-y\left(\frac{m}{\gamma_{\mathrm{BS}} \Omega_{\mathrm{BS}, \mathrm{A}}}-l C D\right)\right) \mathrm{E}_{i}(y l C D),
\end{aligned}
$$


where $C=\frac{\gamma_{\mathrm{RS}} \Omega_{\mathrm{R}, \mathrm{A}}}{\gamma_{\mathrm{BS}} \Omega_{\mathrm{BS}, \mathrm{A}}}$ and $D=\frac{1}{\gamma \Omega_{\mathrm{BS}, \mathrm{R}}}+\frac{1}{\gamma_{\mathrm{RS}} \Omega_{\mathrm{R}, \mathrm{B}}}$. Proof is presented in Appendix.

With this, the sum-rate can be bounded by $\mathcal{R}_{\text {sum }}^{\mathrm{LB}}$ using (1) and bound from (2) as

$$
\begin{aligned}
\mathcal{R}_{\text {sum }} & \geq \frac{1}{2} \log _{2}\left(1+\tilde{\gamma}_{m^{\star}}\right)+\frac{1}{2} \log _{2}\left(1+\gamma_{n, l^{\star}}\right) \\
& \geq \frac{1}{2} \log _{2}\left(1+\tilde{\gamma}_{m^{\star}}\right) \\
& +\frac{1}{2} \log _{2}\left(1+\max _{l \in \mathcal{L}}\left(\frac{1}{2} \min \left(\gamma\left|h_{\mathrm{BS}, l}\right|^{2}, \gamma_{\mathrm{RS}}\left|h_{l, n}\right|^{2} / \mathcal{I}_{n}\right)\right)\right) \\
& =\mathcal{R}_{\mathrm{A}}^{\mathrm{LB}}+\mathcal{R}_{\mathrm{B}}^{\mathrm{LB}}=\mathcal{R}_{\text {sum }}^{\mathrm{LB}}
\end{aligned}
$$

We use the Taylor series method used in literature [16] to bound the ergodic rate. To this end, we first evaluate the moments of the SNRs $\tilde{\gamma}_{m^{\star}}$ and $X=$ $\max _{l \in \mathcal{C}}\left(\frac{1}{2} \min \left(\gamma\left|h_{\mathrm{BS}, l}\right|^{2}, \gamma_{\mathrm{RS}}\left|h_{l, n}\right|^{2} / \mathcal{I}_{n}\right)\right) \leq \gamma_{n, l^{\star}}$. Clearly, the CDF of $X$, similar to (12), is given by

$F_{X}(x)=1-\sum_{l=1}^{L}(-1)^{l-1}\left(\begin{array}{l}L \\ l\end{array}\right) \frac{\exp \left(-2 x l\left(\frac{1}{\gamma \Omega_{\mathrm{BS}, \mathrm{R}}}+\frac{1}{\gamma_{\mathrm{RS}} \Omega_{\mathrm{R}, \mathrm{B}}}\right)\right)}{\frac{l 2 x \gamma_{\mathrm{BS}} \Omega_{\mathrm{BS}, \mathrm{B}}}{\gamma_{\mathrm{RS}} \Omega_{\mathrm{R}, \mathrm{B}}}+1}$.

To obtain $\mathbb{E}[X]$ and $\mathbb{E}\left[X^{2}\right]$, we obtain $t$ th moment of random variable $X\left(\mathbb{E}\left[X^{t}\right]=t \int_{0}^{\infty} x^{t-1}\left(1-F_{X}(x)\right) d x\right)$ using ([17], Eq. (5.53)),[18] as

$$
\begin{aligned}
\mathbb{E}\left[X^{t}\right]= & t \sum_{l=1}^{L} \sum_{j=0}^{t-1}(-1)^{j+l-1}\left(\begin{array}{c}
t-1 \\
j
\end{array}\right)\left(\begin{array}{l}
L \\
l
\end{array}\right) \\
& \times \exp \left(\frac{\gamma_{\mathrm{RS}} \Omega_{\mathrm{R}, \mathrm{B}}}{\gamma \Omega_{\mathrm{BS}, \mathrm{R}} \gamma_{\mathrm{BS}} \Omega_{\mathrm{BS}, \mathrm{B}}}+\frac{1}{\gamma_{\mathrm{BS}} \Omega_{\mathrm{BS}, \mathrm{B}}}\right) \\
& \times\left(\frac{2 l \gamma_{\mathrm{BS}} \Omega_{\mathrm{BS}, \mathrm{B}}}{\gamma_{\mathrm{RS}} \Omega_{\mathrm{R}, \mathrm{B}}}\right)^{-t} \mathrm{E}_{j-t+2}\left(\frac{\gamma_{\mathrm{RS}} \Omega_{\mathrm{R}, \mathrm{B}}}{\gamma \Omega_{\mathrm{BS}, \mathrm{R}} \gamma_{\mathrm{BS}} \Omega_{\mathrm{BS}, \mathrm{B}}}+\frac{1}{\gamma_{\mathrm{BS}} \Omega_{\mathrm{BS}, \mathrm{B}}}\right) .
\end{aligned}
$$

Using the Taylor's series approximation of $\mathbb{E}\left[\frac{1}{2} \log _{2}(1+\right.$ $X)$ ] about the mean, the approximate expression for $\mathbb{E}\left[\mathcal{R}_{\mathrm{B}}^{\mathrm{LB}}\right]$ can be seen to be

$$
\mathbb{E}\left[\mathcal{R}_{\mathrm{B}}^{\mathrm{LB}}\right] \approx \frac{1}{2} \log _{2}(1+\mathbb{E}[X])-\frac{1}{2} \frac{\mathbb{E}\left[X^{2}\right]-(\mathbb{E}[X])^{2}}{\log _{e}(4)(1+\mathbb{E}[X])^{2}}
$$

To determine a bound on the ergodic rate of selected user in cluster $\mathcal{A}$, we again use $\mathbb{E}\left[\tilde{\gamma}_{m^{\star}}^{t}\right]=t \int_{0}^{\infty} x^{t-1}(1-$ $\left.F_{\tilde{\gamma}_{m^{\star}}}(x)\right) d x$ to find the first and second moments of $\tilde{\gamma}_{m^{\star}}$ by using its CDF in (20). The exponential integral appearing in (20) can be rewritten as incomplete Gamma function ([19], Eq. (5.1.45)), after some algebraic manipulation and using ([20], Eq. (6.455)), the closed form expression for the first moment can be shown as

$$
\begin{aligned}
\mathbb{E}\left[\tilde{\gamma}_{m^{\star}}\right] \approx & \sum_{m=1}^{M} \sum_{l=1}^{L} \sum_{i=0}^{m}(-1)^{m+l+i-2}\left(\begin{array}{c}
M \\
m
\end{array}\right)\left(\begin{array}{c}
L \\
l
\end{array}\right)\left(\begin{array}{c}
m \\
i
\end{array}\right) \\
& \times\left[\frac{\gamma_{\mathrm{BS}} \Omega_{\mathrm{BS}, \mathrm{A}}}{m}-i \Gamma(i+1) \times 2 \tilde{F} 1(1,1+i ; 2\right. \\
& \left.\left.+i ; 1-m /\left(\gamma_{\mathrm{BS}} \Omega_{\mathrm{BS}, \mathrm{A}} l C D\right)\right) /(l C D)\right]
\end{aligned}
$$

where $C=\frac{\gamma_{\mathrm{RS}} \Omega_{\mathrm{R}, \mathrm{A}}}{\gamma_{\mathrm{BS}} \Omega_{\mathrm{BS}, \mathrm{A}}}, D=\frac{1}{\gamma \Omega_{\mathrm{BS}, \mathrm{R}}}+\frac{1}{\gamma_{\mathrm{RS}} \Omega_{\mathrm{R}, \mathrm{B}}}$ and ${ }_{2} \tilde{F}_{1}(\cdot, \cdot ; \cdot ; \cdot)$ is regularized Hypergeometric function. Computational software MATHEMATICA can also be used to directly obtain $\mathbb{E}\left[\tilde{\gamma}_{m^{\star}}\right]$ in (25). Similarly, the second moment for SNR of best user in cluster $\mathcal{A}$ is obtained as

$$
\begin{aligned}
\mathbb{E}\left[\tilde{\gamma}_{m^{\star}}^{2}\right] \approx 2 & \sum_{m=1}^{M} \sum_{l=1}^{L} \sum_{i=0}^{m}(-1)^{m+l+i-2}\left(\begin{array}{c}
M \\
m
\end{array}\right)\left(\begin{array}{c}
L \\
l
\end{array}\right)\left(\begin{array}{c}
m \\
i
\end{array}\right) \\
\times & {\left[\frac{\left(l C D-i m /\left(\gamma_{\mathrm{BS}} \Omega_{\mathrm{BS}, \mathrm{A}}\right)\right) l C D}{\left(m /\left(\gamma_{\mathrm{BS}} \Omega_{\mathrm{BS}, \mathrm{A}}\right)\right)^{2}}+i(i+1)\right.} \\
& \times \Gamma(i+2) 2 \tilde{F} 1(1,2+i ; 3+i ; 1 \\
& \left.\left.-m /\left(\gamma_{\mathrm{BS}} \Omega_{\mathrm{BS}, \mathrm{A}} l C D\right)\right)\right] /(l C D)^{2}
\end{aligned}
$$

where $C=\frac{\gamma_{\mathrm{RS}} \Omega_{\mathrm{R}, \mathrm{A}}}{\gamma \Omega_{\mathrm{BS}, \mathrm{R}}}$ and $D=\frac{1}{\gamma \Omega_{\mathrm{BS}, \mathrm{R}}}+\frac{1}{\gamma_{\mathrm{RS}} \Omega_{\mathrm{R}, \mathrm{B}}}$. With this, the rate obtained by user in $\mathcal{A}$ can be written as:

$$
\mathbb{E}\left[\mathcal{R}_{\mathrm{A}}^{\mathrm{LB}}\right] \approx \frac{1}{2} \log _{2}\left(1+\mathbb{E}\left[\tilde{\gamma}_{m^{\star}}\right]\right)-\frac{1}{2} \frac{\mathbb{E}\left[\tilde{\gamma}_{m^{\star}}^{2}\right]-\left(\mathbb{E}\left[\tilde{\gamma}_{m^{\star}}\right]\right)^{2}}{\log _{e}(4)\left(1+\mathbb{E}\left[\tilde{\gamma}_{m^{\star}}\right]\right)^{2}}
$$

Using (24) and (27), in (21) a lower bound on the ergodic rate can be written as

$$
\mathbb{E}\left[\mathcal{R}_{\text {sum }}^{\mathrm{LB}}\right]=\mathbb{E}\left[\mathcal{R}_{\mathrm{A}}^{\mathrm{LB}}\right]+\mathbb{E}\left[\mathcal{R}_{\mathrm{B}}^{\mathrm{LB}}\right] .
$$

Table 1 Outage sub-optimal power allocation factor $\left(\zeta_{\mathrm{BS}}\right)$ for various combinations of $L$ and $M(\{L, M\})$

\begin{tabular}{llllll}
\hline $\boldsymbol{\gamma}$ & $\{\mathbf{1}, \mathbf{3}\}$ & $\{\mathbf{2}, \mathbf{3}\}$ & $\mathbf{\{ 3 , 3 \}}$ & $\mathbf{\{ 3 , 2}\}$ & $\{\mathbf{3}, \mathbf{1}\}$ \\
in $\mathrm{dB}$ & $\boldsymbol{\zeta}_{\mathrm{BS}}$ & $\boldsymbol{\zeta}_{\mathrm{BS}}$ & $\boldsymbol{\zeta}_{\mathrm{BS}}$ & $\boldsymbol{\zeta}_{\mathrm{BS}}$ & $\boldsymbol{\zeta}_{\mathrm{BS}}$ \\
\hline 5 & 0.26 & 0.32 & 0.35 & 0.42 & 0.50 \\
10 & 0.18 & 0.22 & 0.29 & 0.33 & 0.48 \\
15 & 0.11 & 0.17 & 0.22 & 0.26 & 0.40 \\
20 & 0.06 & 0.12 & 0.15 & 0.21 & 0.38 \\
25 & 0.04 & 0.07 & 0.10 & 0.16 & 0.29 \\
\hline
\end{tabular}




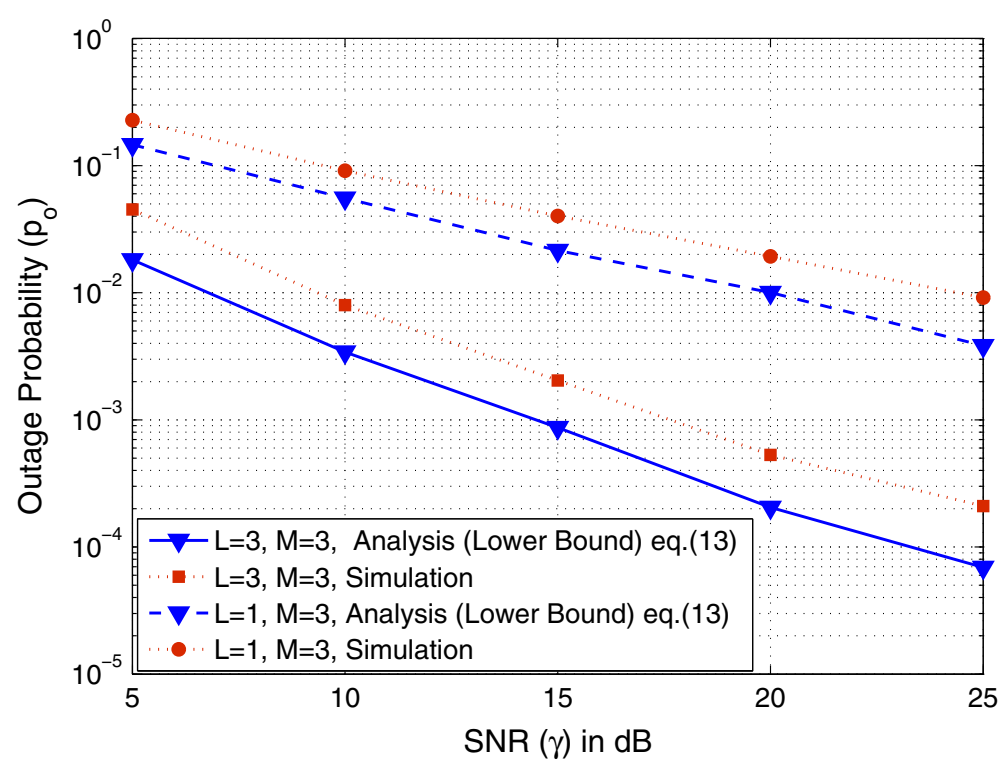

Figure 2 Outage probability $\left(p_{o}\right)$ versus SNR $(\gamma)$ for various number of relays and users combinations.

\section{Special case}

In what follows we discuss the ergodic performance when user selection is applied for users in set $\mathcal{B}$. Exploiting the user scheduling and obtaining the user $n^{\star}$, the ergodic rate for this user is given by

$$
\mathbb{E}\left[\mathcal{R}_{n^{\star}}^{\mathrm{LB}}\right] \approx \frac{1}{2} \log _{2}\left(1+\mathbb{E}\left[X^{\prime}\right]\right)-\frac{1}{2} \frac{\mathbb{E}\left[X^{\prime 2}\right]-\left(\mathbb{E}\left[X^{\prime}\right]\right)^{2}}{\log _{e}(4)\left(1+\mathbb{E}\left[X^{\prime}\right]\right)^{2}}
$$

where

$$
\begin{aligned}
\mathbb{E}\left[X^{\prime t}\right]== & t \sum_{l=1}^{L} \sum_{j=0}^{t-1}(-1)^{j+l-1}\left(\begin{array}{c}
t-1 \\
j
\end{array}\right)\left(\begin{array}{c}
L \\
l
\end{array}\right) \\
& \times \exp \left(\frac{\gamma_{\mathrm{RS}} \Omega_{\mathrm{R}, \mathrm{B}}}{\gamma \Omega_{\mathrm{BS}, \mathrm{R}} \gamma_{\mathrm{BS}} \Omega_{\mathrm{BS}, \mathrm{B}}^{\prime}}+\frac{1}{\gamma_{\mathrm{BS}} \Omega_{\mathrm{BS}, \mathrm{B}}^{\prime}}\right) \\
& \times\left(\frac{2 l \gamma_{\mathrm{BS}} \Omega_{\mathrm{BS}, \mathrm{B}}^{\prime}}{\gamma_{\mathrm{RS}} \Omega_{\mathrm{R}, \mathrm{B}}}\right)^{-t} \mathrm{E}_{j-t+2}\left(\frac{\gamma_{\mathrm{RS}} \Omega_{\mathrm{R}, \mathrm{B}}}{\gamma \Omega_{\mathrm{BS}, \mathrm{R}} \gamma_{\mathrm{BS}} \Omega_{\mathrm{BS}, \mathrm{B}}^{\prime}}+\frac{1}{\gamma_{\mathrm{BS}} \Omega_{\mathrm{BS}, \mathrm{B}}^{\prime}}\right),
\end{aligned}
$$

where $\Omega_{\mathrm{BS}, \mathrm{B}}^{\prime}=\frac{\Omega_{\mathrm{BS}, \mathrm{B}}}{N}, N$ is the number of users in cluster $\mathcal{B}$. As only the statistics of interference channel gain

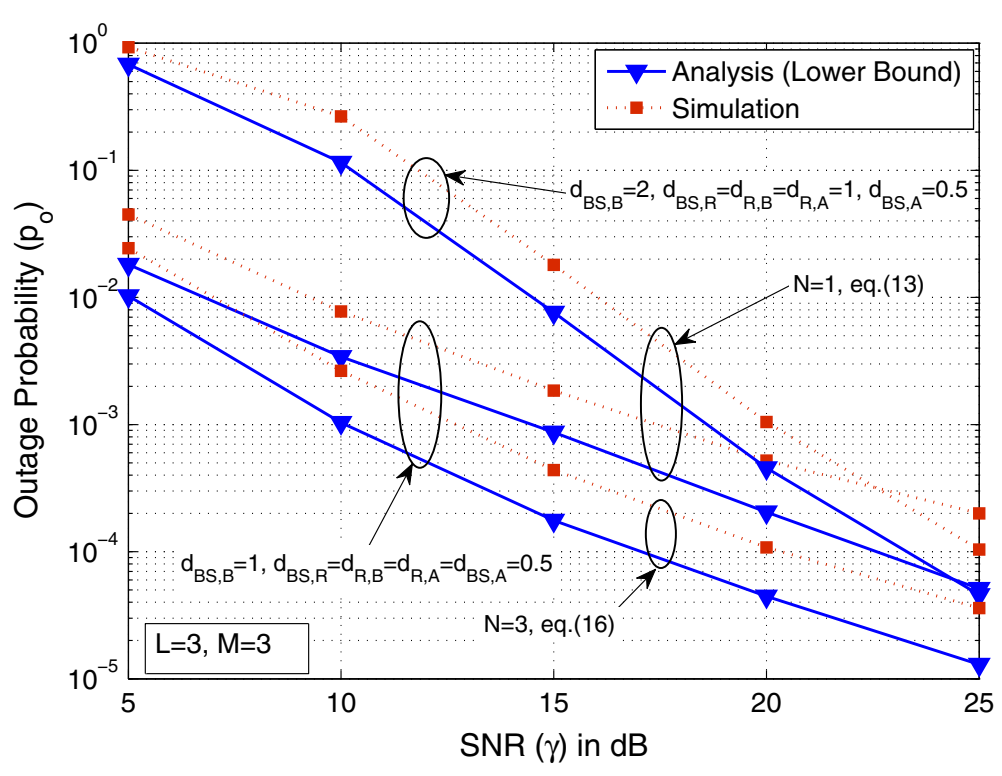

Figure 3 Outage probability $\left(p_{o}\right)$ versus SNR $(\gamma)$ for two different network configurations. 


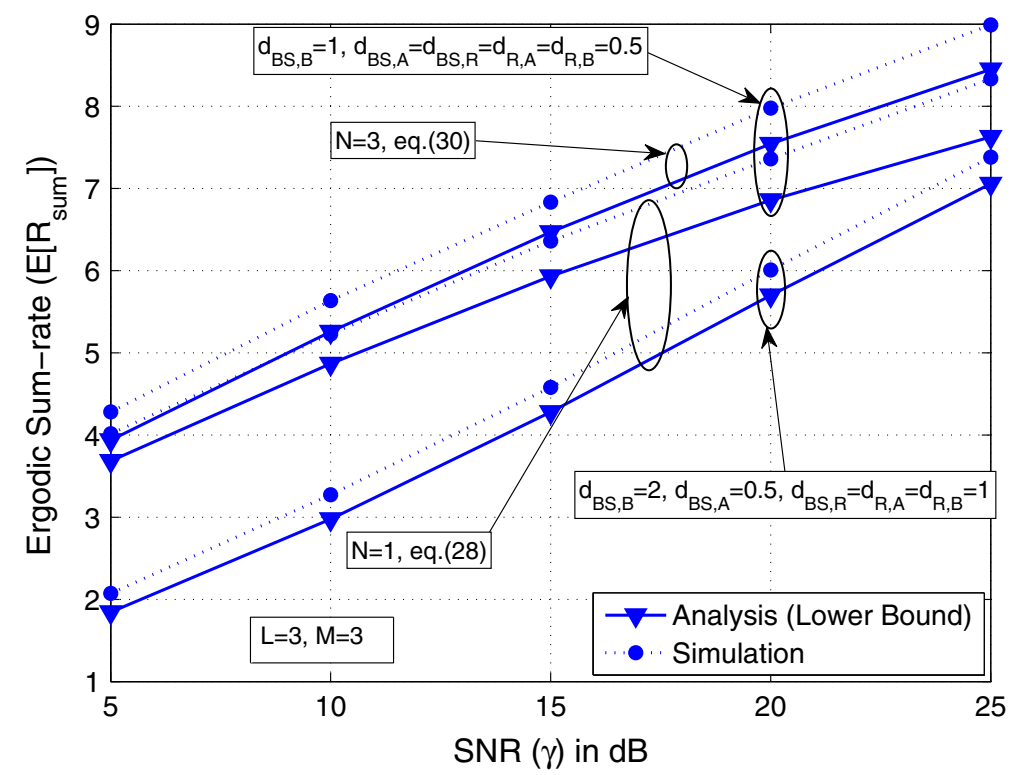

Figure 4 Ergodic sum-rate $\left(\mathbb{E}\left[\mathcal{R}_{\text {sum }}\right]\right)$ versus SNR $(\gamma)$ for two different network configurations.

is changed as given in (15), we can use analysis similar to that used earlier for obtaining the ergodic rate $\mathbb{E}\left[\mathcal{R}_{n^{\star}}^{\prime \mathrm{LB}}\right]$. Resultantly, the ergodic sum-rate is given by

$$
\mathbb{E}\left[\mathcal{R}_{\text {sum }}^{\mathrm{LLB}}\right]=\mathbb{E}\left[\mathcal{R}_{\mathrm{A}}^{\mathrm{LB}}\right]+\mathbb{E}\left[\mathcal{R}_{\mathrm{B}}^{\mathrm{LB}}\right]
$$

As we demonstrate in the next section, the ergodic rate (due to interference cancellation) continuously improves with SNR. Also, the ergodic rate can be improved by increasing $L, M$, and $N$. This makes the use of cooperative multiplexing with user/relay selection advantageous.

\section{Numerical and simulation results}

In this section, we present numerical results based on the derived analytical expressions along with computer simulations, to demonstrate the performance of the considered system. We assume that the normalized distances between nodes $d_{\mathrm{BS}, \mathrm{A}}=d_{\mathrm{BS}, \mathrm{R}}=d_{\mathrm{R}, \mathrm{A}}=d_{\mathrm{R}, \mathrm{B}}=0.5$ with pathloss

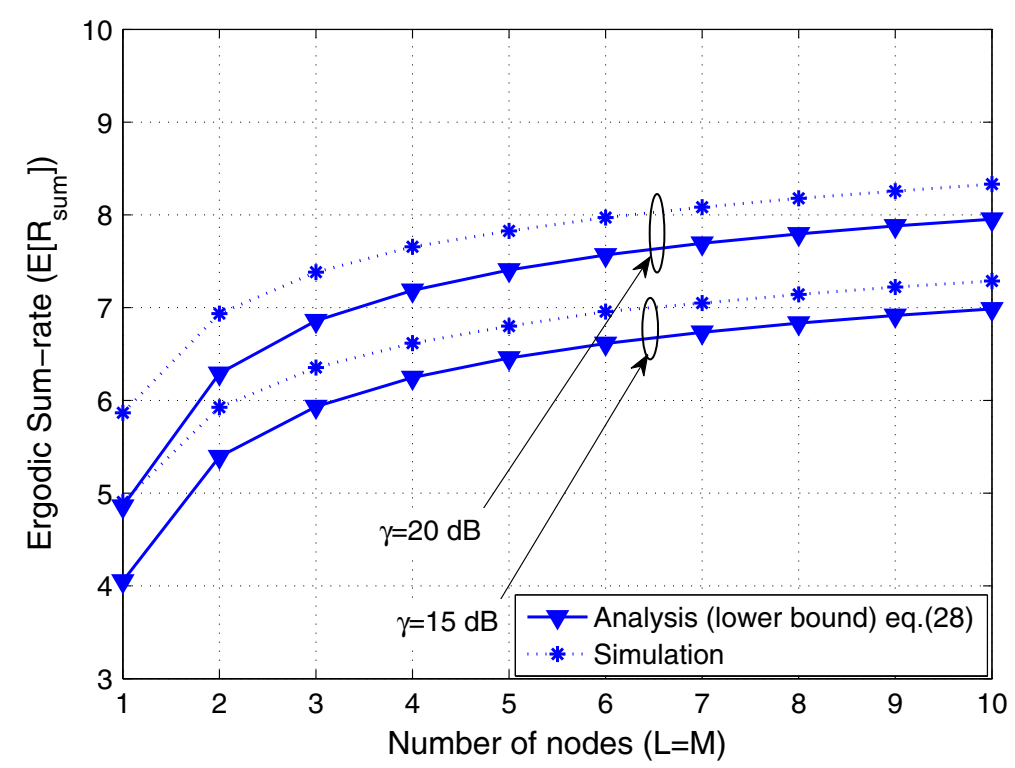

Figure 5 Ergodic sum-rate $\left(\mathbb{E}\left[\mathcal{R}_{\text {sum }}\right]\right)$ of the system versus number of nodes (relay and users). 


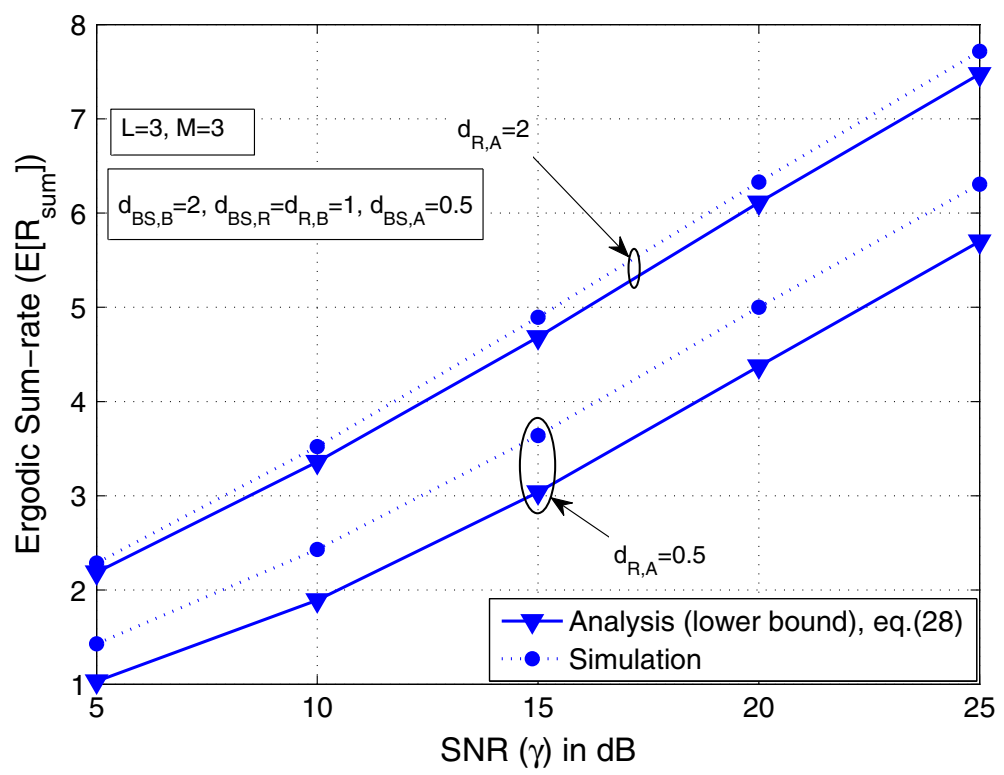

Figure 6 Ergodic sum-rate $\left(\mathbb{E}\left[\mathcal{R}_{\text {sum }}\right]\right)$ versus SNR $(\gamma)$ for two network configurations with different distances between the clusters.

exponent $\alpha=4$, except for the direct channel link from BS to user $\operatorname{set} \mathcal{B}$, which is assumed to be unity $\left(d_{\mathrm{BS}, \mathrm{B}}=1\right)$. We obtain the threshold SNR by considering the target datarate $\mathcal{R}=1 \mathrm{bps} / \mathrm{Hz}$ requirement for both the users. We use (14) to allocate power in the second phase. The optimal power allocation factors $\zeta_{\mathrm{BS}}$ for various parameters are listed in Table 1. From this, it is observed that when the number of users and relays are equal, the power allocation factor is approximately equal, that is quite intuitive for the considered scenario. It is also notable that BS is allocated lesser power than the relay in second phase because of interference from BS to user B.

Figure 2 depicts the outage performance (13) of the system over a range of SNRs $\gamma$ in $\mathrm{dB}$, and shows that the performance of the considered system can be improved by increasing the number of relays and users (which is quite intuitive). In Figure 3, it can be seen that while performance is degraded by increasing the distances between nodes, the crossover at high SNR indicates that performance is improved due to decreased interference at both

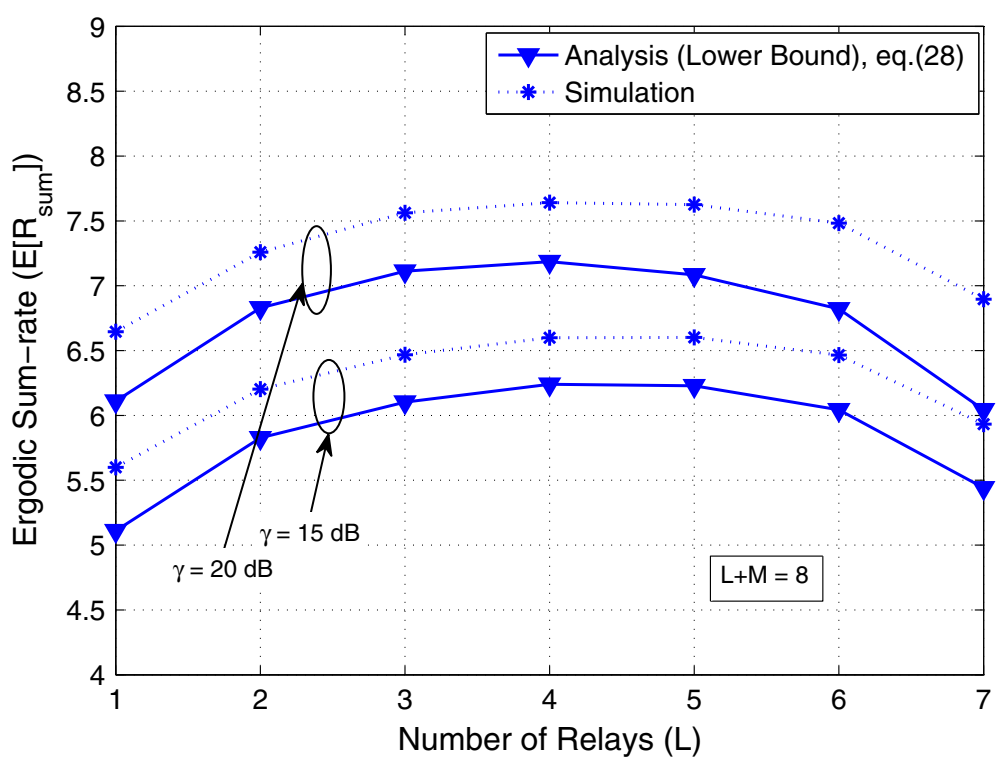

Figure 7 Ergodic sum-rate $\left(\mathbb{E}\left[\mathcal{R}_{\text {sum }}\right]\right)$ versus number of relay nodes ( $L$ ) for fixed number of $L+M$. 
$m^{\star} \in \mathcal{A}$ and $n^{\star} \in \mathcal{B}$. This makes the multiplexing protocol advantageous in large cells. Moreover, we also demonstrate using (16) that scheduling one user $B$ among large number of users is advantageous over arbitrarily consideration of single user.

We now present the ergodic sum-rate of the system in Figures 4 and 5. Using (30), it is demonstrated that the ergodic sum-rate is improved by using scheduling for user in cluster $\mathcal{B}$. It can be seen that the ergodic sum-rate increases with SNR and increase in $L$ and $M$, and this gain diminishes with increasing $L$ and $M$ when these quantities are large. Figure 6 shows the effect of separation of clusters $(\mathcal{A}$ and $\mathcal{L})$. Note that a tighter bound is obtained as the distance between these clusters increases. Figure 7 depicts highest ergodic sum-rate for the equal number relay $(L)$ and user in cluster $(M)$ for fixed number of overall nodes.

\section{Conclusion}

We analyzed the performance of a cooperative spatial multiplexing scheme with opportunistic user and relay selection over Rayleigh fading channels. Bounds were derived for the outage probability and ergodic sumrate with the proposed scheme. A simple power control scheme was suggested to improve outage performance. Performance of the protocol was validated by computer simulations and numerical analysis, and the suggested protocol was shown to be advantageous.

\section{Appendix}

In this section, we derive the CDF expression of $\tilde{\gamma}_{m^{\star}}$. The SNR of best selected user from $\mathcal{A}$ is given by

$$
\tilde{\gamma}_{m^{\star}}=\max _{m \in\{1,2, \ldots, M\}} \tilde{\gamma}_{m}
$$

where $\tilde{\gamma}_{m}=\gamma_{\mathrm{BS}}\left|h_{\mathrm{BS}, m}\right|^{2} \frac{Z}{Z+V}$ where $V=\gamma_{\mathrm{RS}}\left|h_{l^{\star}, m}\right|^{2}$. Using the theory of order statistics, the PDF of $Z$ can be obtained as $f_{Z}(z)=\sum_{l=1}^{L}(-1)^{l-1}\left(\begin{array}{c}L \\ l\end{array}\right) \exp (-z l D)$ where $D=\frac{1}{\gamma \Omega_{\mathrm{BS}, \mathrm{R}}}+$ $\frac{1}{\gamma_{\mathrm{RS}} \Omega_{\mathrm{R}, \mathrm{B}}}$. To obtain the CDF of (31), we obtain $F_{\tilde{\gamma}_{m} \mid Z, V}(y)$ as

$$
F_{\tilde{\gamma}_{m} \mid Z, V}(y)=1-\exp \left(-y \frac{z+v}{z \gamma_{\mathrm{BS}} \Omega_{\mathrm{BS}, \mathrm{A}}}\right)
$$

We first note that $Z$ is common to all the nodes in cluster $\mathcal{A}$. Therefore, we first average over $V$ by using its $f_{V}(v)=$ $\exp \left(-v / \gamma_{\mathrm{RS}} \Omega_{\mathrm{R}, \mathrm{A}}\right) / \gamma_{\mathrm{RS}} \Omega_{\mathrm{R}, \mathrm{A}}$. With this, the conditional CDF is given by

$$
F_{\tilde{\gamma}_{m^{\star}} \mid Z}(y)=1-\sum_{m=1}^{M}(-1)^{m-1}\left(\begin{array}{c}
M \\
m
\end{array}\right) \frac{\exp \left(-m y /\left(\gamma_{\mathrm{BS}} \Omega_{\mathrm{BS}, \mathrm{A}}\right)\right)}{\left(1+\frac{y C}{z}\right)^{m}}
$$

where $C=\frac{\gamma_{\mathrm{RS}} \Omega_{\mathrm{R}, \mathrm{A}}}{\gamma_{\mathrm{BS}} \Omega_{\mathrm{BS}, \mathrm{A}}}$. After averaging by using PDF of $Z$ and using ([19], Eq. (5.1.4)), the CDF of (31) can be obtained as

$$
\begin{aligned}
F_{\tilde{\gamma}_{m^{\star}}}(y)= & 1-\sum_{m=1}^{M} \sum_{l=1}^{L} \sum_{i=0}^{m}(-1)^{m+l+i-2}\left(\begin{array}{c}
M \\
m
\end{array}\right)\left(\begin{array}{c}
L \\
l
\end{array}\right)\left(\begin{array}{c}
m \\
i
\end{array}\right) y l C D \\
& \times \exp \left(-y\left(\frac{m}{\gamma_{\mathrm{BS}} \Omega_{\mathrm{BS}, \mathrm{A}}}-l C D\right)\right) \mathrm{E}_{i}(y l C D) .
\end{aligned}
$$

This completes the proof.

\section{Competing interests}

The authors declare that they have no competing interests.

\section{Acknowledgment}

This study was funded by the Department of Science and Technology, Govt. of India (Project no. SR/S3/EECE/031/2008).

\section{Received: 16 February 2012 Accepted: 27 August 2012}

Published: 21 November 2012

\section{References}

1. J Laneman, D Tse, G Wornell, Cooperative diversity in wireless networks: efficient protocols and outage behavior. IEEE Trans. Inf. Theory. 50(12), 3062-3080 (2004)

2. A Sendonaris, E Erkip, B Aazhang, User cooperation diversity. Part I. System description. IEEE Trans. Commun. 51(11), 1927-1938 (2003)

3. A Sendonaris, E Erkip, B Aazhang, User cooperation diversity. Part II. Implementation aspects and performance analysis. IEEE Trans. Commun. 51(11), 1939-1948 (2003)

4. K Azarian, H El Gamal, P Schniter, On the achievable diversity-multiplexing tradeoff in half-duplex cooperative channels. IEEE Trans. Inf. Theory. $\mathbf{5 1}(12), 4152-4172(2005)$

5. H Yomo, E Carvalho, in IEEE 19th International Symposium on Personal, Indoor and Mobile Radio Communications, 2008. PIMRC 2008. Spectral efficiency enhancement with interference cancellation for wireless relay network, (Cannes, 15-18 September 2008), pp. 1-5

6. B Bandemer, Q Li, X Lin, A Paulraj, in 2009 IEEE 70th Vehicular Technology Conference Fall (VTC 2009-Fall). Overhearing-based interference cancellation for relay networks, (Anchorage, AK, 20-23 September 2009), pp. 1-5

7. CDT Thai, P Popovski, Coordinated direct relay transmission with interference cancelation in wireless systems. IEEE Commun. Lett. 15(4), 416-418 (2011)

8. C Zhong, T Ratnarajah, KK Wong, Outage analysis of decode-and-forward cognitive dual-hop systems with the interference constraint in Nakagami-m fading channels. IEEE Trans. Veh. Technol. 60(6), 2875-2879 (2011)

9. O Oyman, in IEEE International Symposium on Information Theory, 2007. ISIT 2007. Opportunism in multiuser relay channels: scheduling, routing and spectrum reuse, (Nice, 24-29 June 2007), pp. 286-290

10. Y Shi, W Zhang, K Ben Letaief, in IEEE International Conference on Communications, 2008. ICC'08. Cooperative multiplexing and scheduling in wireless relay networks, (Beijing, China, 19-23 May 2008), pp. 3034-3038

11. CK Sung, I Collings, Multiuser cooperative multiplexing with interference suppression in wireless relay networks. IEEE Trans. Wirel. Commun. 9(8), 2528-2538 (2010)

12. A Bletsas, A Khisti, D Reed, A Lippman, A simple Cooperative diversity method based on network path selection. IEEE J. Sel. Areas Commun. 24(3), 659-672 (2006)

13. P Anghel, M Kaveh, Exact symbol error probability of a Cooperative network in a Rayleigh-fading environment. IEEE Trans. Wirel. Commun. 3(5), 1416-1421 (2004)

14. M Chen, T Liu, X Dong, Opportunistic multiple relay selection with outdated channel state information. IEEE Trans. Veh. Technol. 61(3), 1333-1345 (2012)

15. HA David, HN Nagaraja, Order Statistics, 3rd edn. (John Wiley \& Sons, New York, 2003) 
16. D da Costa, S Aissa, Capacity analysis of cooperative systems with relay selection in Nakagami-m fading. IEEE Commun. Lett. 13(9), 637-639 (2009)

17. A Papoulis, S Pillai, Probability, Random Variables and Stochastic Processes, 4th edn. (Tata McGraw-Hill Publishing Company, India, 2002)

18. PL Yeoh, M Elkashlan, I Collings, Selection relaying with transmit beamforming: a comparison of fixed and variable gain relaying. IEEE Trans. Commun. 59(6), 1720-1730 (2011)

19. M Abramowitz, IA Stegun, Handbook of Mathematical Functions with Formulas, Graphs, and Mathematical Tables. (Dover, New York, 1964)

20. IS Gradshteyn, IM Ryzhik, Table of Integrals, Series, and Products, 7th edn. (Academic Press, New York, 2007)

\section{doi:10.1186/1687-1499-2012-345}

Cite this article as: Verma and Prakriya: Performance of a cooperative multiplexing scheme with opportunistic user and relay selection over Rayleigh fading channels. EURASIP Journal on Wireless Communications and Networking 2012 2012:345.

\section{Submit your manuscript to a SpringerOpen ${ }^{\circ}$ journal and benefit from: \\ - Convenient online submission \\ - Rigorous peer review \\ - Immediate publication on acceptance \\ - Open access: articles freely available online \\ - High visibility within the field \\ - Retaining the copyright to your article

